\title{
Monitorización parental de adolescentes con ayuda de la tecnología
}

\author{
Parental monitoring for teenagers using technology \\ Xiomara Paola Carrera Herrera ${ }^{1}$ \\ https://orcid.org/0000-0002-3962-6057 \\ Patricia Maricela Beltrán Guevara ${ }^{2}$ \\ https://orcid.org/0000-0003-4463-9773 \\ Bertha María Villalta Córdova ${ }^{3}$ \\ https://orcid.org/0000-0003-4052-2148 \\ Universidad Técnica Particular de Loja, Ecuador
}

Recibido: 05-01-2020

Aceptado: 14-04-2020

\section{Cita Recomendada}

Carrera, X., Beltrán, P. \& Villalta, B. (2020). Monitorización parental de adolescentes con ayuda de la tecnología. Hamut'ay 7(1), 19-27.

http://dx.doi.org/10.21503/hamu.v7i1.1906

\section{RESUMEN}

La familia se encuentra atravesando algunas transformaciones sociales y los padres actualmente están preocupados por las conductas y las actitudes de sus hijos cuando se encuentran solos. En los tiempos modernos y con la irrupción de las tecnologías es necesario informarse y aprender cómo se deben utilizar estos recursos en las relaciones familiares, de ahí parte la importancia y la necesidad de que los padres de familia hagan conciencia sobre una supervisión más cercana de las actividades de sus hijos, debido a estos antecedentes se ha generado esta investigación, planteando como objetivo principal el identificar el uso de aplicaciones tecnológicas en el proceso de monitorización parental. Para ello, utilizamos un enfoque descriptivo de tipo cuantitativo, el instrumento de aplicación "Entorno familiar y estilo de vida", es un cuestionario Ad hoc dirigido a padres y madres de niños y adolescentes, con una muestra 220 padres de familia, el $75 \%$ madres y $25 \%$ padres, con hijos entre los 11 a 13 ańos de dos instituciones educativas públicas y fiscomisional de la ciudad de Loja, Ecuador. Como conclusiones a la pregunta conoce las actividades que desarrolla su hijo durante el día, el 50\% para la escuela, 15\% para horas de sueño, 7,5\% para actividades de ocio, tiempo libre, actividades artísticas y un segundo idioma; $5 \%$ que están desarrollando actividades con amigos.

Sobre la preocupación parental sobre el uso de la tecnología de los hijos un $50 \%$ de padres señala que como principal problema es que a través de estos medios se hagan amigos de personas desconocidas,

\footnotetext{
1 Docente del Departamento de Ciencias de la Educación, Coordinadora del Grupo de Investigación EDUFAM de la Universidad Técnica Particular de Loja, doctorado en Desarrollo Psicológico, Familia, Educación de la Universidad Santiago de Compostela. xpcarrera@utpl.edu.ec

2 Docente del Departamento de Ciencias de la Educación, Grupo de Investigación EDUFAM, maestría en Gerencia y Liderazgo Educativo por la Universidad Técnica Particular de Loja. pmbeltran@utpl.edu.ec

3 Docente del Departamento de Ciencias de la Educación, Grupo de Investigación EDUFAM, maestría en Pedagogía por la Universidad Técnica Particular de Loja. bmvillalta@utpl.edu.ec
} 
$25 \%$ observen videos de youtubers, un $15 \%$ se agobian por la información sexual que se accedan por las redes sociales y el $10 \%$ por el ciberbullying. Con el Uso del control parental tecnológico para la monitorización los padres de familias desconocen de las herramientas de monitorización parental, pero las redes sociales son más fáciles de identificar por ellos; un $42 \%$ para Facebook, un $31 \%$ WhatsApp, un $17 \%$ a Instagram y $10 \%$ el twitter, preocupante que los padres de familia desconocen de la importancia de dar un seguimiento oportuno a través de herramientas tecnológicas.

Palabras clave: monitorización parental, supervisión, comportamiento, tecnología.

\section{Abstract}

Family is going through some social transformations and parents are currently worried about their children's behavior and attitude when they are alone. Nowadays and with irruption of new technologies it is necessary to be informed and learn how these resources must be used in family relations, from there departs the importance and need that parents raise awareness about a closer supervision from their children's activities, due to this background this research has lead, stating as main objective identifying the use of technological apps in the parental supervising. For this purpose, we use a descriptive approach with quantitative nature, the implementation tool "family surroundings and lifestyle", is a questionnaire Ad hoc directed to children and teenagers' fathers and mothers, with sample of 220 parents, $75 \%$ mothers and $25 \%$ fathers, with children whose ages are from eleven to thirteen years old in two public and Church-state educative institutions in Loja city, Ecuador. As conclusions to the question Do you know the activities your children develop during the day?, $50 \%$ for the school, $15 \%$ for sleeping, $7.5 \%$ for leisure activities, free time, arts and a second language; $5 \%$ are developing activities with friends. About parental concern relating to their children's technology use $50 \%$ parents point as the main problem that through the media they make friends with unknown people, $25 \%$ watch youtubers' videos, $15 \%$ are overwhelmed because of the sexual information their children access through social websites and $10 \%$ for cyberbullying. Relating to the use of technological parental control for monitoring, parents don't know the tools for parental monitoring, but social websites are easier for them to identify; 42\% Facebook, 31\% WhatsApp, $17 \%$ Instagram and $10 \%$ Twitter; it is disturbing that parents don't know the significance of an appropriate monitoring through technological tools.

Keywords: parental monitoring, supervision, behavior, technology.

\section{INTRODUCCIÓN}

La monitorización parental es una dimensión del estilo parental que actualmente ha dado lugar a una serie de investigaciones (Barber, 1996, Delgado, 2005, Keijsers, 2016, Parra \& Oliva, 2006), donde se identifica a esta como un tipo de control conductual por parte de padres y madres que pone límites, reglas y restricciones, así como el modo en que guían y supervisan la conducta de sus hijos mediante una serie de preguntas o su observación, para conocer aspectos de la vida tales como los lugares que frecuentan, o los tipos de amistades y actividades que realizan. Por lo general, estas investigaciones se efectúan en la adolescencia.

La monitorización refleja el esfuerzo de los padres por adaptar y regular la conducta del hijo a través de su guía y supervisión, mientras que el control psicológico emana de una motivación de los padres por seguir manteniendo el poder en la relación, inhibiendo el desarrollo de la autonomía del 
hijo y manteniendo su dependencia de los padres (Pettit \& Laird, 2002, p. 100).

Dentro de este contexto, se ha hecho ineludible hacer un estudio en la relación familiar y la monitorización parental, esto con el propósito de conocer si los padres de familia se preocupan y si se interesan por las diferentes actividades que sus hijos desarrollan en toda su jornada.

$\mathrm{Al}$ analizar el tema de monitorización parental es necesario hacer la reflexión a que refiere este, y podemos decir que es la supervisión de los padres de familia frente a las actividades y conductas de sus hijos; con la modernización a todo nivel, es indispensable que los progenitores acompañen a sus hijos en el proceso de adolescencia, este es una etapa complicada para los jóvenes, en la que afrontan diferentes cambios tanto a nivel físico como psicológico, y lograr que ellos atraviesen esta etapa de una manera adecuada es tarea de sus padres, ellos deben ejercer un acompañamiento efectivo, esto significa que deben ser los responsables en el desarrollo integral.

\section{La monitorización parental}

La familia desempeña aspectos fundamentales para niños y jóvenes, en el cuál debe establecer un crecimiento armónico en lo físico, afectivo, psicológico y moral a partir de un clima de afecto, estimulación, protección y supervisión, fomentando la comunicación y la práctica de valores basados en principios de igualdad, logrando así un desarrollo general. La monitorización parental o supervisión de los padres es una extensión del estilo parental que actualmente ha dado lugar a una serie de investigaciones (Barber, 1996, Delgado, 2005, Parra \& Oliva, 2006), siendo la monitorización parental un tipo de control conductual por parte de padres y madres para establecer límites, reglas y restricciones, así como el modo en que guían y supervisan la conducta de sus hijos mediante una serie de preguntas o su observación, para conocer aspectos de la vida tales como los lugares que frecuentan, o los tipos de amistades y actividades que realizan.

La supervisión demuestra el esfuerzo de los padres por adaptar y regular hacia una conducta positiva del hijo a través de su guía y supervisión, mientras que el control psicológico emana de una motivación de padres por seguir manteniendo el poder en la relación, inhibiendo el desarrollo de la autonomía del hijo y protegiendo su dependencia de los padres (Pettit \& Laird, 2002, p. 100). Por el contrario, Kerr et al., (2003) hacen referencia a varios estudios que encontraron relacionado entre la comprensión que los padres tienen de las actividades de sus hijos y el grado de ajuste comportamental, lo cual no obedecería al hecho de que la monitorización parental impida el surgimiento de comportamientos inadecuados, sino que más bien sería debido al hecho de que la información que poseen los progenitores procede de la auto-revelación de sus hijos, y son los adolescentes más ajustados y con menos problemas conductuales quienes tienden a contar más cosas a sus padres.

Desde otras perspectivas, Dishion \& McMahon (1998) lo define como: "un grupo de comportamientos parentales correlacionados, que implican atención a y seguir la pista de los hijos e hijas acerca de sus lugares de salida, actividades y adaptaciones" (p. 61). Los motivos que evidencian la importancia del control para el desarrollo y ajuste del adolescente tienen que ver por un lado con la estructura que proporciona, y que poco a poco sería interiorizada por el adolescente, y por otro, con que los esfuerzos de seguimiento y monitorización serían necesarios para mantener a jóvenes alejados de las amistades conflictivas y de los problemas de conducta.

La monitorización parental también puede ser identificada como un tipo de control conductual, ampliamente definida, es una habilidad parental relevante desde la infancia a la adolescencia e incluso en los inicios de la adultez, que otorga mayor autonomía al adolescente y permite, junto con la auto-revelación, conseguir conocimiento parental de su hijo o hija: acerca de sus actividades, relaciones con iguales y lugares de salida (Smetana, Campione-Barr, et al., 2006). Pastor, Martín \& Montes (2018) refieren que el control parental se realiza más a las hijas, con la preocupación que estas puedan hablar con desconocidos por el temor del acoso sexual, y la edad es otro aspecto preponderante, siendo la etapa de la adolescencia en la enseñanza secundaria la que tiene un mayor control. 
Los niños y adolescentes actualmente se encuentran vinculados a la tecnología y muchas veces es imposible negarlo, la gran cantidad de dispositivos que usan estos es mayor cada vez, y los padres no pueden supervisar cada momento que estén utilizándolos, allí es donde las herramientas de control parental logran ser pertinentes, existen software que nos ofrecen la capacidad de bloquear contenido web no deseado, limitar el tiempo de pantalla, geolocalización y el restringir el uso de aplicaciones riesgosas, entre otras; estos servicios son una forma positiva de proteger a los hijos y estén más seguros en el uso de estos medios.

\section{Modelos de monitorización parental}

Existen algunos modelos en el campo de la monitorización parental, entre ellos tenemos a $\mathrm{Di}$ shion \& McMahon (1998) quienes propusieron un modelo tríadico, que cuenta con tres dimensiones parentales dinámicamente interrelacionadas, relevantes para la prevención: a) Motivación, representa el sistema de creencias parental (el marco socio- cognitivo), incluyendo normas, valores y metas parentales, muy relevantes e influyentes en las prácticas parentales; b) Monitorización parental, seguir la pista y estructurar las actividades y los contextos de género y c) Conducta directiva, intentos parentales activos que posibilitan resultados positivos en chicos y chicas, como el uso de incentivos, reforzamiento positivo, creación de límites y negociación. En la que se pueden mencionar, tres dimensiones que están incorporadas dentro de las relaciones parento-filiales y se influyen mutuamente para favorecer relaciones positivas.

Otro modelo importante; es el propuesto por $\mathrm{Ha}-$ yes, Hudson \& Matthews (2003) que une todos los elementos de las relaciones parentales conceptualizadas como un proceso interactivo y dinámico entre padres, adolescentes y el contexto. Este modelo comprende una serie de elementos: a) Evaluación del comportamiento de los progenitores y del adolescente; b) Hipótesis sobre la función de sus comportamientos y su proceso cíclico; c) Una evaluación de las características parentales que contribuyen a las interacciones de monitorización; d) Una evaluación de las características del adolescente; e) La interacción del contexto familiar con otros contextos (iguales, escuela, comunidad, cultura, área geográfica y socioeconómica).

Como tercer modelo relevante en el estudio de la monitorización parental, tenemos al de Kerr et al., (2003), quienes plantearon un modelo de interacciones de carácter bidireccional del conocimiento parental, donde se sugieren que padres y madres muestren interés y ejerzan control parental sobre su adolescente. Como conclusión expusieron que el tiempo que los adolescentes pasan fuera de la supervisión de sus padres se desarrolla la confianza parental.

Como cuarto modelo Fletcher, Steinberg \& Williams-Wheeler (2004) plantearon dos modelos alternativos que permiten reformular investigaciones anteriores: el primero donde señala que las relaciones afectivas de los jóvenes se manifiestan espontáneamente brindando información a sus padres. El segundo modelo sostiene que las relaciones indirectas de los padres presentan consecuencias en los problemas de conducta del adolescente.

Desde estas teorías sobre los modelos de monitorización parental no cabe duda que la principal finalidad de los padres es el bienestar de los hijos en esta sociedad tan colmada de problemas sociales, morales, académicos y tecnológicos.

\section{La tecnología en la adolescencia}

La generación actual tiene la capacidad de interactuar de distintas formas utilizando una variedad de escenarios tecnológicos al mismo tiempo, los adolescentes son los que han hecho de esta tecnología su estilo de vida al haber nacido y crecido en esta era tecnológica, al usar las mismas como un medio social, educativo, de ocio y como vinculo de relaciones amicales (Alvites-Huamaní, 2019), además los adolescentes han impuesto al Internet como un recurso fundamental para sus relaciones sociales, Álvarez-García, García, Cueli \& Núñez, (2018), aspectos de los cuales no están exentos los jóvenes del Ecuador, debido a que han desarrollado características que no difieren en forma significativa de sus pares de otros continentes, y los riesgos y amenazas que se han ido incorporando, hace a los jóvenes y adolescentes presa fáciles de muchas ciberadicciones y personas sin escrúpulos, lo importante es observar y analizar como los protagonistas 
asocian cada una de estas herramientas y a la vez los peligros que se reflejan cuando los adolescentes se escudan tras una pantalla y no saben cómo relacionarse con su entorno.

Barrio Fernández \& Fernández (2016) señalan que la brecha digital existente entre padres e hijos, con gran desconocimiento en muchos casos por parte de ellos relacionado con la informática y las posibles medidas de control y prevención, dificulta aún más el proceso educativo y de orientación y seguimiento.

La utilización de la tecnología por niños y jóvenes es sumamente habitual en todos los hogares, y ha dado lugar a importantes transformaciones en el uso del tiempo libre, el aprendizaje, la exploración, la socialización e incluso la afectividad, perturbando como consecuencia a las relaciones interpersonales. Se han llevado a cabo nuevos estudios sobre los efectos de las redes sociales virtuales con población infantil y adolescente, pero realmente ¿Qué hacen tanto tiempo en Internet? ¿Qué personas conforman sus redes sociales? Y otras interrogantes que se nos vienen al momento de observar el tiempo dedicado a Internet. Gross (2004) puntualiza en su estudio que la población juvenil destina el uso a tres actividades básicas en el ámbito privado: (1) revisar mensajes de correo electrónico e instantáneos, (2) relación con amigos que también son parte de sus vidas diarias, fuera de línea, y (3). dedicación a temas íntimos (por ejemplo, los amigos, el chisme) (p. 646).

Por consiguiente, en los jóvenes se ha comprobado que han comunicado intimidades y dan a conocer sus sentimientos más profundos a través de las redes sociales virtuales, mientras que en forma física estos mismos jóvenes tienden a ser silenciosos y a mantener conversaciones superficiales, limitando la verdadera comunicación sólo al espacio virtual. Al mismo tiempo se ha encontrado que los jóvenes y nińos que presentan problemas en sus hogares son más propensos a comunicarse con personas extrañas y a compartir sus problemas como forma de desahogarse (Subrahmanyam \& Greenfield, 2008).

El uso de la tecnología debe ser tutelado por los padres con una orientación, observación y dirección debida para el uso adecuado de las TIC, combinando estas actividades con formas de es- parcimiento que proporcionen un equilibrio en el desarrollo de los niños y adolescentes, con el fin de evitar que los hijos caigan en desenlaces negativos, producto de una escasa guía paterna.

\section{MATERIALES Y MÉTODOS}

\section{Participantes}

Esta investigación es una prueba piloto que se desarrolló en la provincia de Loja, la población estuvo constituida por una muestra no probabilística de conveniencia o disponibilidad (Etikan, Musa \& Alkassim, 2016) el cual se eligió a 220 padres de familia de dos instituciones educativas, de estudiantes del $7^{\circ}, 8^{\circ}$ y $9^{\circ}$ año de educación básica de escuelas fiscales y fiscomisionales.

\section{Instrumento}

Para la investigación realizada se desarrolló un instrumento Ad hoc "Entorno familiar y estilo de vida", dirigido a padres de familia; este cuestionario se estructura sobre la relación familiar y el estilo de vida donde se incluye la monitorización parental con el uso de la tecnología, los aspectos que se consideran en este estudio son los propios de la monitorización parental y el uso de la tecnología. Para la confiabilidad del instrumento se logró encontrar un elevado grado de confiabilidad con un Alfa de Cronbach de .86 para 20 ítems.

\section{Tipo y Diseño}

Para esta investigación se utilizó un enfoque descriptivo de tipo cuantitativo (Real, 2016). Este enfoque permite estar cerca del fenómeno estudiado a la realidad, y nos provee de un sentido de comprensión más completo, sin intentar relacionar las diferentes variables analizadas, ni buscar explicaciones a la encontrado.

\section{Procedimiento}

La investigación se realizó en cinco etapas, las cua- 
les se estructuraron de la siguiente manera:

\section{Etapa:}

Diagnóstico realizado mediante el proyecto " $\mathrm{Fa}$ milia Felices" dirigido a padres de dos instituciones educativas, en el cual se pudo detectar problemas en las relaciones con los hijos adolescentes.

\section{Etapa:}

Establecimiento de objetivos y variables mediante la contextualización de las principales problemáticas existentes en los jóvenes y padres de familia.

\section{Etapa:}

Búsqueda de información bibliográfica con información de trabajos realizados en la temática.

\section{Etapa:}

Operacionalización de las variables que se definirán y medirán el estudio.

\section{Etapa:}

Elaboración del cuestionario, validación por expertos, aplicación de la prueba piloto a los padres de familia, y análisis de resultados.

\section{Confidencialidad y consentimiento informado:}

La investigación se hizo de manera anónima, con la firma del consentimiento informado voluntario de los participantes,, explicando los objetivos de la investigación, considerando que los participantes deben ser conscientes de su derecho a negarse a participar; y explicando que se mantendrá la confidencialidad

\section{Resultados}

A continuación, presentamos algunos descriptivos relevantes en esté estudio piloto, con respecto a la información socio demográfica de la muestra investigada, el estudio se realizó a padres de familia conformados por $75 \%$ de madres y $25 \%$ de padres. La edad de los hijos de padres investigados fluctúa de 11 a 13 años, con un nivel de instrucción de los padres predominante en el nivel superior con un $45 \%$, además los tipos de familia son: nuclear con un $45 \%$, seguida por la extensa con $32 \%$, la monoparental con un $9 \%$ y la compuesta el $1 \%$.

\section{Tabla 1}

Actividades de los adolescentes.

\begin{tabular}{lccccc}
\hline $\begin{array}{l}\text { Usted conoce de } \\
\text { las actividades } \\
\text { que desarrolla } \\
\text { su hijo durante } \\
\text { el día }\end{array}$ & $\begin{array}{c}\text { Muy fre- } \\
\text { cuente }\end{array}$ & $\begin{array}{c}\text { Fre- } \\
\text { cuente }\end{array}$ & $\begin{array}{c}\text { Ocasio- } \\
\text { nalmente }\end{array}$ & $\begin{array}{c}\text { Rara } \\
\text { vez }\end{array}$ & Nunca \\
\hline $\begin{array}{l}\text { Escuela } \\
\text { Actividades ex- }\end{array}$ & 0 & 30 & 0 & 0 & 0 \\
tracurriculares. & 0 & 30 & 0 & 0 & 0 \\
\hline $\begin{array}{l}\text { Actividades de } \\
\text { ocio y tiempo }\end{array}$ & 0 & 0 & 0 & 0 \\
libre & 0 & 0 & 10 & 0 & 0 \\
\hline $\begin{array}{l}\text { Actividades } \\
\text { con el grupo de } \\
\text { amigos }\end{array}$ & & & & & \\
\hline $\begin{array}{l}\text { Horas de sueño } \\
\text { Actividad física }\end{array}$ & 40 & 0 & 0 & 0 & 0 \\
\hline
\end{tabular}

Fuente: Elaboración propia (2020)

Usted conoce las actividades que desarrolla su hijo durante el dia

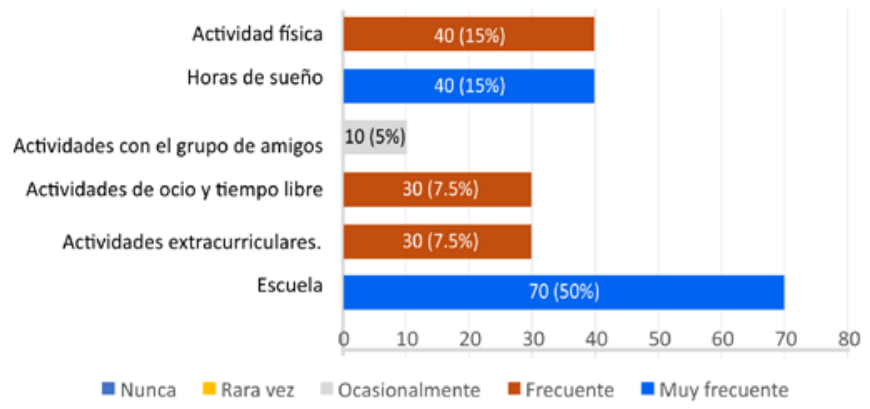

\section{Figura 1}

Actividades de los adolescentes.

Fuente: Elaboración propia (2020)

Al momento de analizar los resultados obtenidos en la primera tabla y de acuerdo a la interrogante planteada, usted conoce de las actividades que desarrolla su hijo durante el día se pudo rescatar en estos primeros resultados que la mitad de la muestra investigada (50\%) de los padres de familia manifiestan que sus hijos están en la escuela, un 15\% de los progenitores señalan que sus hijos destinan a horas de sueño, dos resultados semejantes de un $7,5 \%$ manifiestan que sus hijos se involucran en actividades de ocio y tiempo libre, así como también actividades extra curriculares como: deporte, 
actividades artísticas y un segundo idioma; un porcentaje menor del $5 \%$ de padres mencionan que están desarrollando actividades con un grupo de amigos.

\section{Tabla 2}

Preocupación parental sobre el uso de la tecnología de los hijos.

\begin{tabular}{|c|c|c|c|c|c|}
\hline $\begin{array}{l}\text { Usted conoce de } \\
\text { las actividades } \\
\text { que desarrolla } \\
\text { su hijo durante } \\
\text { el día }\end{array}$ & $\begin{array}{l}\text { Muy fre- } \\
\text { cuente }\end{array}$ & $\begin{array}{l}\text { Fre- } \\
\text { cuente }\end{array}$ & $\begin{array}{l}\text { Ocasio- } \\
\text { nalmente }\end{array}$ & $\begin{array}{l}\text { Rara } \\
\text { vez }\end{array}$ & Nunca \\
\hline $\begin{array}{l}\text { Información } \\
\text { sexual }\end{array}$ & 0 & 0 & 40 & 0 & 0 \\
\hline Cyberbuling. & 0 & 30 & 0 & 0 & 0 \\
\hline $\begin{array}{l}\text { Videos De You- } \\
\text { tuber Negativos }\end{array}$ & 0 & 0 & 0 & 50 & 0 \\
\hline $\begin{array}{l}\text { Amigo de per- } \\
\text { sonas descono- } \\
\text { cidas }\end{array}$ & 80 & 0 & 0 & 0 & 0 \\
\hline
\end{tabular}

Fuente: Elaboración propia (2020)

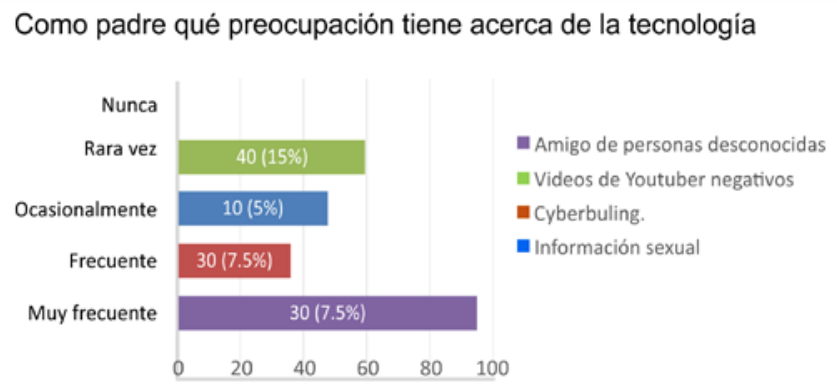

Figura 2

Preocupación acerca de la tecnología.

Fuente: Elaboración propia (2020)

Como padres la preocupación que tienen acerca de la implicación de la vida de sus hijos con el uso de la tecnología, un 50\% de ellos señala que como principal problema es que a través de estos medios se hagan amigos de personas desconocidas, otro porcentaje importante del $25 \%$ les inquieta que observen videos de youtubers con contenidos negativos que propicien en ellos ideas distorsionadas, un $15 \%$ se agobian por la información sexual que sus hijos puedan acceder a través de las redes sociales; y finalmente el ciberbullying se presenta con un $10 \%$.

\section{Tabla 3}

Uso del control parental tecnológico para la monitorización.

\begin{tabular}{lcc}
\hline $\begin{array}{l}\text { Conoce usted alguna de las siguientes } \\
\text { aplicaciones }\end{array}$ & Si & No \\
\hline Spyzie & & 0 \\
\hline Kids Place & 0 \\
\hline Webwatcher & 0 \\
\hline Qustodio & 0 \\
\hline Norton Family Premier & & 0 \\
\hline Facebook & 200 & \\
\hline WhatsApp & 150 & \\
\hline Instagram & 80 & \\
\hline Twitter & 50 & \\
\hline
\end{tabular}

Fuente: Elaboración propia (2020)

Uso del control parental tecnológico para la monitorización

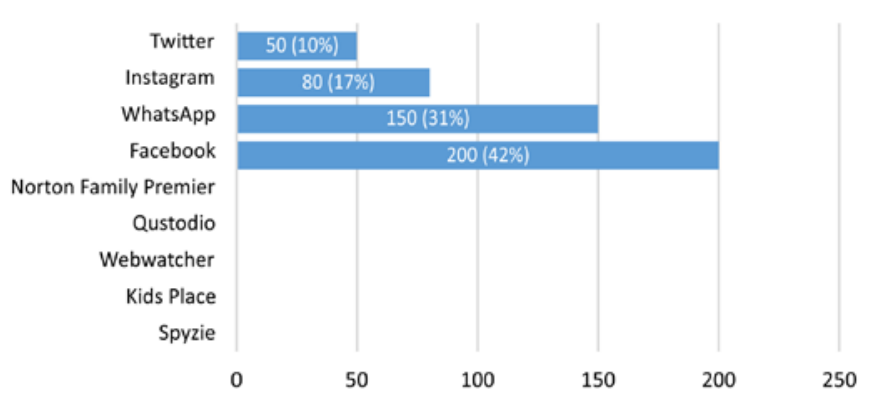

Figura 3

Control parental tecnológico.

Fuente: Elaboración propia (2020)

Algo muy interesante al hacer este estudio es conocer sobre el uso del control parental tecnológico para la monitorización, una de las preguntas planteadas más relevantes de nuestra investigación fue fusionar las herramientas tecnológicas con las redes sociales, esto permitió identificar que los padres de familias desconocen de las herramientas de monitorización parental, sin embargo las redes sociales son más fáciles de identificar por ellos; dando así como resultado un 42\% para Facebook, un 31\% WhatsApp, un $17 \%$ a Instagram y con el $10 \%$ el twitter. Estos resultados nos permiten comprobar que los padres de familia desconocen de la importancia de dar un seguimiento oportuno a través de herramientas tecnológicas. 


\section{Discusión y Conclusiones}

La monitorización parental debe ser vista como un factor protector en el desarrollo del comportamiento del adolescente, este proceso de intervención de los padres está fuertemente influenciado por la comunicación padre-hijo y su calidad en la relación. El uso de la tecnología debe ser tutelado por los padres con una orientación, observación y dirección debida para el uso adecuado de las TIC, combinando con actividades de esparcimiento que proporcionen un equilibrio en el desarrollo de los niños y adolescentes, con el fin de evitar que los hijos caigan en desenlaces negativos, producto de una escasa guía paterna. Los adolescentes deben ser conscientes de la necesidad que tienen los padres sobre el proceso de monitorización considerando el respeto a la autonomía (Crouter \& Head, 2002; Marshall, Tilton-Weaver \& Bosdet, 2005;).

Los resultados han demostrado que los adolescentes no poseen un control parental riguroso en las actividades diarias y del uso de las nuevas tecnologías, por la falta de tiempo de los padres e insuficiente atención en las problemáticas que actualmente vive la sociedad, en relación algunas investigaciones señalan que los progenitores deben ser modelos para el uso de la tecnología de sus hijos (Aoyama, Utsumi, \& Hasegawa, 2012; Sarah, Vaala \& Bleakley, 2015). Además, las prácticas generales de los padres que no son específicas de los medios de comunicación también pueden afectar el comportamiento mediático de los jóvenes. Por ello como grupo de investigación se sugiere que los padres de familia utilicen el diálogo y recursos tecnológicos que permitan conocer las actividades y las conductas de sus hijos frente a sus grupos sociales.

La monitorización con ayuda de las herramientas tecnológicas, permitirán ser un recurso educativo que involucrará poseer información y capacidad para estructurar de forma activa el contexto en el que los hijos desarrollan su estilo de vida (juego, salidas con amigos, actividades sociales y culturales, entre otras). Es fundamental indicar que la monitorización debe adaptarse a las características evolutivas de la conducta de niños y adolescentes. Así, la monitorización en etapas infantiles debe ajustarse más en los ámbitos del hogar y la escuela, mientras que en la adolescencia estos ámbitos son más amplios incluyendo: redes de amigos, redes sociales, lugares de ocio, parejas afectivas.

\section{Agradecimiento}

Un agradecimiento especial a los estudiantes Camilo Mesa, Leído Malaver, Álvaro Sánchez; Juan José Sáenz, Julieta Paternina, Shaila Jiménez; quienes conforman el Semillero de Investigación "Duendes Matemáticos", quienes participaron de manera activa en la investigación siendo estudiantes de quinto de primaria Colegio Nueva Zelandia IED, Suba, Colombia.

\section{REFERENCIAS BIBLIOGRÁFICAS}

Álvarez-García, D., García, T., Cueli, M. \& Núñez, J. (2018). Control parental del uso de internet durante la adolescencia: Evolución y diferencias de género. Revista Iberoamericana de Diagnóstico y Evaluación, 51 (2) 19-31. https://doi.org/10.21865/RIDEP51.2.02

Alvites-Huamaní, C. G. (2019). Adolescencia, ciberbullying y depresión riesgos en un mundo globalizado. Revista científica electrónica de Educación y Comunicación en la Sociedad del Conocimiento, 19 (1), 210-234. Recuperado de http://eticanet.org/revista/index.php/eticanet/article/ view/182/159

Aoyama, I., Utsumi, S. \& Hasegawa, M. (2012). Ciberacoso en Japón: casos, informes gubernamentales, agresión relacional en adolescentes y funciones de supervisión parental.

Barber, C. B., Dobkin, D. P., Dobkin, D. P., \& Huhdanpaa, H. (1996). The quickhull algorithm for convex hulls. ACM Transactions on Mathematical Software (TOMS), 22(4), 469-483. https://doi.org/10.1145/235815.235821

Barrio Fernández, Á., \& Fernández, I. R. (2016). Los adolescentes y el uso de las redes sociales. International Journal of Developmental and Educational Psychology. Revista INFAD de Psicología., 3(1), 571-576.

https://doi.org/10.17060/ijodaep.2014.n1.v3.537

Crouter, AC y Head, MR (2002). Control parental y conocimiento de los niños. En M. Bornstein (Ed.), Handbook of parenting, 2nd ed., Vol. 3: Convertirse y ser padre (págs. 461 - 483). Mahwah, Nueva Jersey: Erlbaum.

Delgado, A. O. (2005). Relaciones familiares y desarrollo adolescente. Anuario de psicología, 36(3).

Dishion, T. J., \& McMahon, R. J. (1998). Parental monitoring and the prevention of child and adolescent problem behavior: A conceptual and empirical formulation. Clinical 
child and family psychology review, 1(1), 61-75.

https://doi.org/10.1023/A:1021800432380

Gross, E. F. (2004). Adolescent Internet use: What we expect, what teens report. Journal of Applied Developmental Psychology, 633- 649.

https://doi.org/10.1016/j.appdev.2004.09.005

Kerr, M., Stattin, H., Biesecker, G., \& Ferrer- Wreder, L. (2003). Relationships with parents and peers in adolescence. Handbook of psychology, 395-419.

https://doi.org/10.1002/0471264385.wei0616

Marshall, SK, Tilton-Weaver, LC y Bosdet, L. (2005). Gestión de la información: considerando la regulación del conocimiento parental por parte de los adolescentes. Diario de la Adolescencia, 28, 633- 647.

https://doi.org/10.1016/j.adolescence.2005.08.008

Parra, Á., \& Oliva, A. (2006). Un análisis longitudinal sobre las dimensiones relevantes del estilo parental durante la adolescencia. Infancia y Aprendizaje, 29(4), 453-470.

https://doi.org/10.1174/021037006778849594

Pettit, G. S., \& Laird, R. D. (2002). Psychological control and monitoring in early adolescence: The role of parental involvement and earlier child adjustment.

https://doi.org/10.1037/10422-004

Sarah E. Vaala \& Amy Bleakley (2015) Monitoreo, mediación y modelado: influencia de los padres en el uso de computadoras e Internet en adolescentes en los Estados Unidos, Journal of Children and Media, 9 (1), 40-57. https://doi. org/10.1080 / 17482798.2015.997103

Pastor, Y., Martín, R. \& Montes, Y. (2018). Patrones de uso, control parental y acceso a la información de los adolescentes en la red. Revista Estudios. 25(2), 9951012. https://revistas.ucm.es/index.php/ESMP/article/ view/64821/4564456551630

Smetana, J. G., Campione-Barr, N., \& Metzger, A. (2006). Adolescent development in interpersonal and societal contexts. Annu. Rev. Psychol., 57, 255-284.

https://doi.org/10.1146/annurev.psych.57.102904.190124

Subrahmanyam, K., \& Greenfield, P. (2008). Online communication and adolescent relationships. The future of children, 18(1), 119-146.

https://doi.org/10.1353/foc.0.0006 